\title{
Cardiología y Geriatría en la práctica diaria: un reto hacia el futuro
}

\section{Cardiology and Geriatrics in daily practice: a challenge for the future}

\author{
Luis H. García Ortiz ${ }^{a, b, c}$
}

\author{
a Departamento de Medicina Interna, Facultad de Ciencias de la Salud, Universidad Tecnológica de Pereira, Pereira, Colombia \\ b Fundación Cardiomet, Pereira, Colombia \\ c Centro de Investigaciones Clínicas IPS, Pereira, Colombia
}

Recibido el 25 de agosto de 2016; aceptado el 26 de agosto de 2016

Disponible en Internet el 13 de septiembre de 2016

"¡Qué desgracia!, exclamó el príncipe, que los seres humanos embriagados por el orgullo propio de la juventud, no vean la vejez ;Volvamos rápido a casa! De qué sirven los juegos y las alegrías si soy la morada de la futura vejez"'.

Príncipe Sidarta Buda ${ }^{1}$

En la historia de la humanidad siempre han sido un mito la inmortalidad y la juventud eterna. Historias como las de Matusalén y Dorian Gray o lugares maravillosos como Shangri-La donde las personas no morían y vivían toda la eternidad, siempre serán tema de libros y películas, seguro exitosas. Este fenómeno existe en todas las culturas y religiones. Siempre vamos a temer envejecer y morir.

El envejecimiento es un fenómeno universal, progresivo e irreversible. Los avances médicos y tecnológicos han logrado que la expectativa de vida hoy supere los 80 años y que sea más frecuente encontrar centenarios en nuestra población.

La población geriátrica puede dividirse en viejos (75/84 años) y muy viejos (85 y más). En Colombia, como país en vía de desarrollo, se considera paciente geriátrico a aquel mayor de 60 años, adulto joven hasta los 60 años y adulto mayor después de esta edad; sin embargo, el grupo de mayores de 80 años es el de mayor crecimiento actual proporcionalmente. La clasificación de la American Geria-

Correo electrónico: estudioscardiomet@gmail.com tric Society (AGS) tiene en cuenta además a los más viejos (oldest) y centenarios (ancients).

No obstante, estos avances importantes también tienen consecuencias, entre ellas, mayores costos sociales y económicos. Si la edad de jubilación hoy es en promedio 62 años, significa que vamos a pasar casi un $20 \%$ de nuestro resto de vida sin ser productivos económicamente y si no tenemos un proyecto de vida a futuro, es un tiempo "eterno"', durante el cual y por cambios asociados al envejecimiento, tendremos mayor probabilidad de padecer enfermedades crónicas y de alto costo.

En la encuesta Nacional de envejecimiento de 2016 en Colombia, el $11 \%$ de nuestra población corresponde a mayores de 60 años y de ellos el 60\% trabaja porque necesita el dinero, el $58 \%$ lo hace de manera informal y apenas el $29 \%$ recibe pensión.

La pirámide poblacional está invirtiéndose y ante la menor tasa de natalidad y mayor senescencia, la proporción de mayores será cada vez más alta. La primera causa de morbilidad y mortalidad hoy son las enfermedades cardiovasculares.

La edad como factor de riesgo a partir de los 45 años se explica porque los cambios asociados a esta, como pérdida de elastina vascular, disfunción endotelial, elastocalcinosis entre muchos otros fenómenos normales del envejecimiento, que aumentan la resistencia vascular, la postcarga y por ende la rigidez de los vasos y estructuras vasculares, válvulas cardiacas y tractos de 
conducción explican la alta prevalencia de hipertensión arterial sistólica, bloqueos AV, arritmias y enfermedad arteriosclerótica, y son además la evidencia de la etiología cardiovascular como primera causa de mortalidad hoy en esta población ${ }^{3}$.

Aparte de la edad como factor de riesgo, acumulamos desde la infancia actividades no saludables como sedentarismo, mal uso del tiempo libre y malos hábitos alimenticios, muy influenciados por los medios de comunicación y la cultura del consumo fácil, las comidas rápidas, el exceso de tecnología como los videojuegos, el uso de controles remotos para todo, los ascensores para pisos bajos, entre tantos gadgets que ofrece la modernidad.

De otra parte, las variables sociales y el rol de la mujer que cambió para bien de ellas, también impacta en el hogar como núcleo principal de los buenos hábitos. Los niños reciben la educación "integral" en los colegios, allí se alimentan (¿sano?) y realizan actividades lúdicas y deportivas (?); aprenden a ser cómodos y a que todo sea rápido.

La obesidad ligada a malos hábitos desde la infancia (epigenética negativa), que seguro se debe a todos estos cambios, ya inició una cascada de eventos fisiopatológicos que se expresarán en la edad adulta joven.

Bien se sabe que la inflamación persistente de bajo grado asociada a la obesidad, produce cambios acelerados en la vasculatura sistémica; pero esos cambios no son medibles con exámenes de rutina (interleukinas, TNF, PCR HS, metaloproteinasas). A edad temprana todo es asintomático. Aun no se detecta hipertensión, dislipidemia o diabetes y menos aterosclerosis. Son niños "sanos" 4 .

En un adulto joven (menor de 40 años y asintomático) posiblemente el score de calcio arterial por doppler muestre daño arterial subclínico ${ }^{5,6}$. Infortunadamente no se realizan valoraciones de prevención primaria en edades más tempranas porque la educación clásica dada a la población consiste en "ir al médico cuando estoy enfermo" y la educación médica se basa en enfermedades visibles e intervenciones invasivas; prevención secundaria.

Así, la situación nos lleva hoy a encontrar personas mayores con inflamación vascular crónica no diagnosticada y que aunque no hayan sufrido eventos finales cardiovasculares (infarto agudo de miocardio, enfermedad cerebrovascular, enfermedad arterial periférica oclusiva, enfermedad renal crónica), conforman hoy un porcentaje importante de nuestra sociedad: los "barrigones", o sea la obesidad sarcopénica por envejecimiento, sedentarismo y malos hábitos nutricionales.

Ese personaje gordito y "asintomático" es candidato protagonista para todos los eventos finales cardiovasculares y todas las intervenciones farmacológicas e invasivas que tenemos hoy en nuestras guías de manejo.

Es importante tener en cuenta que la inflamación vascular persistente de bajo grado por obesidad sarcopénica, además produce cambios en vasos de la subcorteza cerebral que explican a mediano plazo deterioro cognitivo leve, trastornos de la marcha y depresión que a su vez expresan la mayor vulnerabilidad del anciano. Uno de los temores más grandes al envejecer es perder la memoria y ser dependientes.

Cuando todo lo anterior es utópico y no se alcanzó una prevención primaria óptima ni un control adecuado de factores de riesgo para un envejecimiento saludable y exitoso, nos enfrentamos, como sucede hoy, al paciente anciano vulnerable en el servicio de urgencias, la unidad de cuidado intensivo, la sala de hospitalización o la consulta.

Los retos clínicos al evaluar un paciente anciano son grandes y una recomendación importante es realizar una valoración geriátrica integral, la cual constituye una manera de evaluar el soporte social y económico, la capacidad funcional y la independencia para las actividades de la vida diaria, el estado cognitivo y afectivo, además de las comorbilidades diagnosticadas o no, que influenciarán el pronóstico a corto plazo. Un paciente con demencia subclínica, síndrome depresivo atípico del anciano, dependiente para sus actividades básicas y con deterioro de la función renal aun con creatinina en rango normal (nefrosclerosis asociada al envejecimiento), debe ser detectado previamente $\mathrm{y}$ antes de procedimientos invasivos para definir metas y propuestas terapéuticas adecuadas.

Conocer la farmacocinética es importante al iniciar o modificar la terapia farmacológica. La absorción, biodisponibilidad y unión a proteínas, así como el volumen de distribución, varían durante el proceso de envejecimiento. La polifarmacia es muy prevalente en este grupo. Un paciente anciano con enfermedad coronaria e insuficiencia cardiaca, ya se enfrenta como mínimo a recibir hasta cinco medicamentos, hecho que incrementa el riesgo de sangrado, hipotensión arterial, incontinencia urinaria, trastornos hidroelectrolíticos y riesgo de caídas. Al usar anticoagulantes es imperativo tener presentes las interacciones medicamentosas. En general, además de medicaciones para otras patologías crónicas (EPOC, artritis reumatoide, diabetes) que deben ser tratadas de forma individual, debemos tener en cuenta las medicaciones de venta libre y automedicadas, que aumentan el riesgo de delirium.

Los eventos adversos medicamentosos en ancianos que reciben más de cinco fármacos, son mayores al 50\% y representan una causa importante de ingreso y estancias hospitalarios.

La fragilidad es uno de los grandes síndromes geriátricos y esencialmente aumenta el riesgo de morbilidad y mortalidad por todas las causas en pacientes mayores de 65 años. Se caracteriza por pérdida de la reserva celular, tisular y fisiológica que aumenta el riesgo de complicaciones ante cualquier estresor.

Los criterios de fragilidad son de fácil detección y difícil interpretación. Es básicamente un alerta del paciente más vulnerable ${ }^{7}$.

Los criterios de Fried hacen diagnóstico de fragilidad con tres de cinco de ellos:

- Pérdida de peso no intencional.

- Sentimiento de agotamiento general.

- Debilidad (medida por fuerza de agarre).

- Lentitud de la marcha (basados en una distancia de 4,6 m).

- Bajo nivel de actividad física (menos de 400 Cal $x$ semana).

Un anciano frágil no diagnosticado previamente que se hospitaliza por cualquier causa, tiene mayor riesgo de complicación y muerte por este fenotipo frágil que las dadas por los riesgos inherentes a las patologías de base de cualquier etiología. 
Recientemente se han publicado artículos de consenso sobre el anciano frágil en diferentes patologías ${ }^{7,8}$.

Para el diagnóstico y tratamiento de la hipertensión arterial en muy ancianos, las guías son conflictivas ${ }^{9}$ y estudios recientes involucran ya al menos el criterio de fragilidad.

De igual forma, no existe consenso para el tratamiento del síndrome coronario agudo en ancianos frágiles; individualizar es el argumento aceptado.

A futuro se espera que sea cada vez más frecuente enfrentar nonagenarios y centenarios activos y vitales e independientes. En este grupo, sin discusión, es preciso ofrecer todos los avances médicos, farmacológicos e intervencionistas, obviamente tratando de prevenir daños y optimizando estrategias terapéuticas.

Una valoración adecuada del paciente anciano que incluya todas estas recomendaciones, evitará complicaciones, acortará la estancia hospitalaria y mejorará la calidad de vida de estos y sus familias. El valor social del anciano en la sociedad actual y en su núcleo familiar, es muy alto.

"Una bella ancianidad es ordinariamente la recompensa de una bella vida".

(Pitágoras)

\section{Bibliografía}

1. De Beauvoir S. La vielleisse. Primera edición. México: Editorial Hermes; 1983.
2. Beard JR, Officer AM, Cassels AK. The world report on ageing and health. Gerontologist. 2016;56 Suppl 2:S163-6.

3. Wang M, Monticone RE, Lakatta EG. Proinflammation of aging central arteries: a mini-review. Gerontology. 2014;60: 519-29.

4. Fuster V, Sanz J. Vascular inflammation. J Am Soc Hypertens. 2007;1:68-81.

5. Yaffe K, Vittinghoff E, Pletcher MJ, Hoang TD, Launer LJ, Whitmer $\mathrm{R}$, et al. Early adult to midlife cardiovascular risk factors and cognitive function. Circulation. 2014;129:1560-7.

6. Baber U, Mehran R, Sartori S, Schoos MM, Sillesen H, Muntendam $\mathrm{P}$, et al. Prevalence, impact, and predictive value of detecting subclinical coronary and carotid atherosclerosis in asymptomatic adults: the Biolmage study. J Am Coll Cardiol. 2015;65: 1065-74.

7. Fried LP, Walston J. Frailty and failure to thrive. En: Hazzard WR, Blass JP, Ettinger WH Jr, Halter JB, Oslander J, editores. Principles of Geriatric Medicine and Gerontology. $5^{\text {th }}$. ed. New York: Mc Graw Hill; 2003. p. 1487-502.

8. Benetos A, Bulpitt CJ, Petrovic M, Ungar A, Agabiti Rosei E, Cherubini $A$, et al. An expert opinion from the European Society of Hypertension-European Union Geriatric Medicine Society Working Group on the Management of Hypertension in Very Old, Frail Subjects. Hypertension. 2016;67:820-5.

9. Psaltis PJ, Nicholls SJ. Management of acute coronary syndrome in the very elderly. Lancet. 2016;387(10023):1029-30. 